\title{
A DECOMPOSITION OF ELEMENTS OF THE FREE ALGEBRA
}

\author{
WENXIN MA
}

(Communicated by Maurice Auslander)

\begin{abstract}
Let $f$ be an element of $F[X]$, the free associative algebra over a field $F$ and $n$ the maximum of the degrees of the variables and the multiplicities of the degrees in $f$. A partial ordering on the homogeneous elements of $F[X]$ is defined such that if $f$ is homogeneous and char $F \nmid n$ !, then $f$ can be decomposed into a sum of two polynomials $f_{0}$ and $f_{1}$ such that for $0<m \leq n, f_{0}$ is symmetric or skew symmetric in all its arguments of degree $m$ depending on whether $m$ is even or odd and $f_{1}$ is a consequence of polynomials of lower type than $f$. Osborn's Theorem about the symmetry of the absolutely irreducible polynomial identities is obtained as a corollary. The same holds in the free nonassociative algebra. The proofs are combinatorial.
\end{abstract}

\section{INTRODUCTION}

In [2] Osborn introduced a partial ordering on the free nonassociative algebra $F\langle X\rangle$ over a field $F$ and proved the following interesting result: if $n$ is a positive integer with char $F \nmid n$ ! and $f$ is an identity of a not necessarily associative algebra $A$ over $F$ such that $A$ has no identity of type lower than $f$ in the partial ordering, then $f$ is symmetric or skew symmetric in its arguments of degree $n$ depending on whether $n$ is even or odd. This theorem was used in [1] to determine the identities of degree $2 n$ of the space of $n \times n$ symmetric matrices; however, the assumption that $A$ has no identity of lower type limits the use of the result in many cases. In this paper we remove the restriction that $A$ has no identities of lower type and consider polynomials at large, not only polynomial identities. Osborn's Theorem follows as a consequence.

Throughout the paper $F$ always denotes a field. The polynomials are in the free associative algebra $F[X]$, where $X$ is a set of countable noncommuting indeterminates $x_{1}, x_{2}, \ldots$ over the field $F$. All identities mentioned are homogeneous weak identities (i.e., polynomials that evaluate to zero on some fixed subspace $V$ of an algebra $A$ ) except when otherwise noted.

\section{A PARTIAL ORDER, $\Delta$-OPERATORS, AND WEAK $T$-IDEALS}

Following Osborn $[2$, p. 78] we introduce a partial ordering on the set of homogeneous polynomials in $F[X]$, the free associative algebra in the noncommuting variables $X=\left\{x_{1}, x_{2}, \ldots\right\}$. If $p\left(x_{1}, \ldots, x_{m}\right)$ is a homogeneous

Received by the editors May 17, 1991 and, in revised form, August 26, 1991.

1991 Mathematics Subject Classification. Primary 16A38. 
polynomial of degree $n$, as in [3], we say that $p$ is of type $\left[n_{1}, \ldots, n_{m}\right]$ if $n_{j}$ is the degree of $x_{j}$ in $p$ and $n_{m} \neq 0$ but $n_{j}=0$ for $j>m$. Define $\left[\left[n_{1}, \ldots, n_{m}\right]\right]$ to be $\left[n_{i_{1}}, \ldots, n_{i_{m}}\right]$, where $n_{i_{1}} \geq n_{i_{2}} \geq \cdots \geq n_{i_{m}}$ and $\left\{n_{i_{1}}, \ldots, n_{i_{m}}\right\}=\left\{n_{1}, \ldots, n_{m}\right\}$ as multisets. Let $p^{\prime}$ be a homogeneous polynomial of degree $n^{\prime}$ and of type $\left[n_{1}^{\prime}, \ldots, n_{m^{\prime}}^{\prime}\right]$. If

$$
\left[\left[n_{1}^{\prime}, n_{2}^{\prime}, \ldots, n_{m^{\prime}}^{\prime}\right]\right]=\left[n_{j_{1}}^{\prime}, n_{j_{2}}^{\prime}, \ldots, n_{j_{m^{\prime}}}^{\prime}\right]
$$

then $p$ is lower than $p^{\prime}$ in the partial ordering if and only if either (i) $n<n^{\prime}$ or (ii) $n=n^{\prime}$ and $n_{i_{k}}>n_{j_{k}}^{\prime}$ for the first integer $k$ such that $n_{i_{k}} \neq n_{j_{k}}^{\prime}$; otherwise, the two polynomials are not comparable. If an integer $n_{j}$ is repeated $k$ times, we shall denote this by an exponent; for example, $\left[3,2^{2}, 1^{3}\right]$ means $[3,2,2,1$, $1,1]$.

Let $f\left(x_{1}, x_{2}, \ldots, x_{i}, \ldots, x_{j}, \ldots, x_{m}\right) \in F[X]$ be a homogeneous polynomial and $x_{i}, x_{j}$ have degree $n_{i}, n_{j}$ in $f$, respectively. Then the polynomial

$$
\begin{aligned}
f^{\prime}\left(x_{1}, \ldots, x_{i}, \ldots, x_{j}, \ldots, x_{m}\right) \\
\quad:=f\left(x_{1}, \ldots, x_{i}+x_{j}, \ldots, x_{j}, \ldots, x_{m}\right) \\
\quad=f\left(x_{1}, \ldots, x_{i}, \ldots, x_{j}, \ldots, x_{m}\right)+f_{1}+\cdots+f_{n_{i}},
\end{aligned}
$$

where $f_{k}$ is the homogeneous component of $f^{\prime}$ and $x_{i}, x_{j}$ have degree $n_{i}-k$, $n_{j}+k$ in $f_{k}$, respectively. Each $f_{k}$ is obtained by setting $k x_{i}$ 's to be $x_{j}$ in $f$. Following [3, p. 9] we define

$$
\Delta^{k}\left(x_{i}, x_{j}\right) f:=f_{k}, \quad k=1,2, \ldots, n_{i}, \quad \Delta^{0}\left(x_{i}, x_{j}\right) f:=f .
$$

The mapping $\Delta^{k}$ is called a $\Delta$-operator (or a derivation) and $\Delta^{k}\left(x_{i}, x_{j}\right) f$ is called a partial linearization of $f$. If $n_{i}>0$ and $n_{j}=0$ (which is allowed in the above definition) then $\Delta^{1}\left(x_{i}, x_{j}\right) f$ is of degree $n_{i}-1$ in $x_{i}$ and 1 in $x_{j}$. Therefore, if enough variables of degree 0 were present then one could linearize $f$ using successive $\Delta$-operators. We denote by $\Delta f$ the set of all polynomials in $F[X]$ that can be obtained from $f$ by means of repeated $\Delta$-operations

$$
\Delta f=\left\{g \in F[X] \mid g=\Delta^{j_{1}}\left(x_{i_{1}}, x_{k_{1}}\right) \cdots \Delta^{j_{s}}\left(x_{i_{s}}, x_{k_{s}}\right) f\right\} .
$$

It is well known that the set $T(A)$ of all polynomial identities of an $F$-algebra is a $T$-ideal, i.e., an ideal of $F[X]$ that is invariant under all endomorphisms of $F[X]$. However, this is no longer true for weak identities. Hence we introduce the concept of weak $T$-ideals.

Definition 1. An ideal $W$ of $F[X]$ is called a weak $T$-ideal if $W$ is invariant under every linear mapping $x_{i} \mapsto \sum \alpha_{i j} x_{j}$ for $\alpha_{i j} \in F$.

If $S$ is a subset of $F[X]$ then the smallest weak $T$-ideal containing $S$ in $F[X]$ is called the weak $T$-ideal generated by $S$ and denoted by $\langle S\rangle$.

One basic example of weak $T$-ideal is the ideal $T(A, V)$ consisting of all weak polynomial identities on a subspace $V$ of an algebra $A$.

Using a standard Vandermonde argument (e.g., [3, Theorem 5, p. 12]) one can prove

Proposition 1. Let $f\left(\ldots, x_{i}, \ldots, x_{j}, \ldots\right) \in F[X]$ be a homogeneous polynomial of type $\left[\ldots, n_{i}, \ldots, n_{j}, \ldots\right]$. If $|F|>n_{i}$ then $\Delta^{k}\left(x_{i}, x_{j}\right) f \in\langle f\rangle$, 
$k=0,1, \ldots, n_{i}$. In particular, if $f \in T(A, V)$ then $\Delta^{k}\left(x_{i}, x_{j}\right) f \in T(A, V)$ for $k=0,1, \ldots, n_{i}$.

Let $f\left(x_{1}, \ldots, x_{n}\right)$ and $g\left(x_{1}, \ldots, x_{n}\right)$ be homogeneous polynomials of $F[X]$. We shall say that $g$ is a consequence of (or comes from) the polynomial $f$ if $g$ belongs to the vector space $\operatorname{Span}\{\Delta f\}$.

Since a partial linearization of an identity $f$ is not necessarily an identity, the polynomials in $\Delta f$ need not be identities either. But from Proposition 1, if $F$ contains enough elements then $\Delta f$ lies in the $T$-ideal $\langle f\rangle$, and hence if $g$ comes from $f$ then $g$ is a linear combination of identities in $\langle f\rangle$.

\section{THE MAIN RESULTS}

As in [3], the free associative algebra $F[X]$ has a decomposition into a direct sum of subspaces $V^{\left[n_{1}, \ldots, n_{k}\right]_{[}}[X]$ consisting of the homogeneous polynomials of type $\left[n_{1}, \ldots, n_{k}\right]$. In this section we give a decomposition of the elements of $V^{\left[n_{1}, \ldots, n_{k}\right]}[X]$, which is useful in the study of identities.

Theorem 1. Let $n$ be a positive integer and let $p$ be a homogeneous polynomial with coefficients in a field $F$ of characteristic not dividing $n$ !. Let $x$ and $y$ be arguments of degree $n$. Define $p_{1}$ by

$$
p_{1}:=p(\ldots, y, \ldots, x, \ldots)-(-1)^{n} p(\ldots, x, \ldots, y, \ldots) .
$$

Then the following statements hold:

(1) The polynomial $p_{1}$ comes from a polynomial of lower type than $p$.

(2) Let $\operatorname{char} F \neq 2$. If there exist two variables in $p$, either of even degree in which $p$ is skew symmetric or of odd degree in which $p$ is symmetric, then $p=\frac{1}{2} p_{1}$ comes from a polynomial of lower type.

(3) If $|F|>2 n-1$ and $p$ is an identity, then $p_{1}$ is a linear combination of identities of type lower than $p$.

(4) If $\Delta(y, x) p=0$ then $p$ is either symmetric or skew symmetric in $x$ and $y$ depending on whether $n$ is even or odd.

Proof. Define

$$
\tilde{p}_{1}:=\alpha_{0} f_{n}+\alpha_{1} f_{n-1}+\cdots+\alpha_{n-1} f_{1},
$$

where $\alpha_{k}=(-1)^{k} k ! / n(n-1) \cdots(n-k), 0 \leq k \leq n-1$,

$$
\begin{aligned}
f_{1}:= & \Delta^{0}(y, x) \Delta^{1}(x, y) \Delta^{1}(y, x) p, \\
f_{2}:= & \Delta^{1}(y, x) \Delta^{2}(x, y) \Delta^{2}(y, x) p-\left(\begin{array}{c}
n \\
1
\end{array}\right) f_{1}, \\
f_{m+1}:= & \Delta^{m}(y, x) \Delta^{m+1}(x, y) \Delta^{2}(x, y) p \\
& -\left(\begin{array}{c}
n \\
m
\end{array}\right) f_{1}-\left(\begin{array}{c}
n-1 \\
m-1
\end{array}\right) f_{2}-\cdots-\left(\begin{array}{c}
n-m+1 \\
1
\end{array}\right) f_{m} .
\end{aligned}
$$

The polynomial $\tilde{p}_{1}$ comes from $\Delta^{1}(y, x) p$, which is of lower type than $p$.

If $|F|>2 n-1$ and $p$ is an identity, then for each $i, i=1, \ldots, n$, $f_{i} \in\left\langle\Delta^{1}(y, x) p\right\rangle$ by Proposition 1 and hence $\tilde{p}_{1}$ is a linear combination of 
consequences of lower type identities $f_{i}$. Thus, to prove statements (1) and (3) of the theorem, it suffices to show that

$$
p(\ldots, y, \ldots, x, \ldots)=(-1)^{n} p(\ldots, x, \ldots, y, \ldots)+\tilde{p}_{1} .
$$

Since $\Delta^{k}(x, y)$ and $\Delta^{m}(y, x)$ change only the variables $x$ and $y$, the polynomials $p(\ldots, y, \ldots, x, \ldots),(-1)^{n} p(\ldots, x, \ldots, y, \ldots)$, and $\tilde{p}_{1}$ are of the same type. So, they are linear combinations of the same monomials. Thus, to show (3) it suffices to show that for each monomial $M$, the coefficient of $M$ in $p(\ldots, y, \ldots, x, \ldots)$ and the coefficient of $M$ in $(-1)^{n} p(\ldots, x, \ldots, y, \ldots)+$ $\tilde{p}_{1}$ are the same.

First we define an equivalence relation on the set of monomials occurring in $p: M_{1} \sim M_{2}$ if $M_{2}$ is obtainable from $M_{1}$ by permuting some $x$ 's and $y$ 's and leaving the other variables fixed. We denote by $[M]$ the equivalence class of $M$. Let $I$ be the set of all associative words in $x$ and $y$ involving exactly $n x$ 's and $n y$ 's. Setting the variables, other than $x$ and $y$, equal to 1 in an element of $[M]$ yields a bijection from $[M]$ to $I$; so we may write $[M]=\left\{M_{i} \mid i \in I\right\}$ and, in particular, $M=M_{w}$ for some $w \in I$. Let $I^{(k)}, k=-1,0,1, \ldots, n$, be the set of all associative words that have $n+k \quad y$ 's and $n-k x$ 's. Then $I=I^{(0)}$ and $I^{\prime}:=I^{(-1)}$ is the set of all associative words in $x$ and $y$ involving exactly $n+1 x$ 's and $n-1 y$ 's. For $i, j \in I^{\prime} \cup I \cup I^{(1)} \cup \cdots \cup I^{(n)}$, we define $i \cdot j$ to be the number of positions in which both $i$ and $j$ have a $y$.

Let $c_{i}$ be the coefficient of $M_{i}$ in $p$ and $r_{j}$ be the coefficient of $M_{j}$ in $p^{\prime}:=\Delta^{1}(y, x) p$. Then for each $j \in I^{\prime}$ the total coefficient of $M_{j}$ in $p^{\prime}$ will be the sum of the $n+1$ coefficients $c_{i}$ where $i$ runs over all those elements of $I$ such that $i \cdot j=n-1$, i.e.,

$$
r_{j}=\sum_{i \bullet j=n-1} c_{i} \forall j \in I^{\prime}
$$

If $k=j \cdot w$ then every $i$ occurring in (4) satisfies either $i \cdot w=k$ or $i \cdot w=k+1$. Indeed since $j$ has $n-1$ elements $y$ 's and $i \cdot j=n-1, i$ has a $y$ in a given position whenever $j$ does. So in the positions where both $j$ and $w$ have a $y$, $i$ has a $y$ also. Therefore $j \cdot w=k$ implies $i \cdot w$ is at least $k$. But $i$ has only one more $y$ than $j$, so $i \cdot w \leq k+1$. Each $i \in I$ satisfying $i \cdot w=k$ occurs in exactly $n-k$ equations of type (4) for which $j \cdot w=k$. Indeed $i$ occurs in (4) iff $M_{i}$ becomes $M_{j}$ when replacing one $y$ by an $x$ in $M_{i}$; since $i \cdot w=k$, if we put one $y$ in $M_{i}$ that occurs in the same position in $w$ to be $x$ to get $M_{j}$, then $j \cdot w=k-1$ not $k$. This cannot happen, so we have only $n-k$ choices and each one produces distinct $M_{j}$ 's, so $c_{i}$ occurs $n-k$ times in the set of equations of type (4) satisfying $j \cdot w=k$. Each $i \in I$ satisfying $i \cdot w=k+1$ occurs in exactly $k+1$ equations of type (4) satisfying $j \cdot w=k$, for, this time, we can only choose those $y$ 's that occur in the same position in $i$ and $w$ in order to get $j \cdot w=k$.

Now we add up all equations of type (4) satisfying $j \cdot w=k$ to get

$$
\sum_{j \cdot w=k} r_{j}=(n-k) C_{k}+(k+1) C_{k+1}, \quad k=0,1,2, \ldots, n-1,
$$

where, for $0 \leq m \leq n, C_{m}:=\sum_{i \cdot w=m} c_{i}$, with $c_{i}$ occurring in an equation of type (4) satisfying $j \cdot w=k$. Substituting the $n$ equations of (5) into one 
another yields

$$
\begin{aligned}
C_{0}= & (-1)^{n} C_{n}+(-1)^{n-1} \frac{(n-1) !}{n !} \sum_{j \cdot w=n-1} r_{j}+(-1)^{n-2} \frac{(n-2) !}{n(n-1) \cdots 2} \\
& \times \sum_{j \cdot w=n-2} r_{j}+\cdots-\frac{1}{n(n-1)} \sum_{j \cdot w=1} r_{j}+\frac{1}{n} \sum_{j \cdot w=0} r_{j} .
\end{aligned}
$$

We know that $C_{n}=c_{w}$ and $C_{0}=c_{w^{\prime}}$, where $w^{\prime}$ is the word obtained from $w$ by interchanging all the $x$ 's and $y$ 's. Thus we have that

$$
c_{w^{\prime}}=(-1)^{n} c_{w}+\alpha_{n-1} \beta_{n-1}+\cdots+\alpha_{0} \beta_{0},
$$

where $\alpha_{k}=(-1)^{k} k ! / n \cdots(n-k)$ and $\beta_{k}=\sum_{j \cdot w=k} r_{j}, k=0,1, \ldots, n-1$. Next we show that $\sum_{i=0}^{n-1} \alpha_{i} \beta_{i}$ is the coefficient of the monomial $M_{w}$ in $\tilde{p}_{1}$ and, therefore, $\tilde{p}_{1}$ comes from $p^{\prime}$. By the definition of $\tilde{p}_{1}$, it suffices to show that each $\beta_{n-k}$ is the coefficient of $M_{w}$ in $f_{k}$. This will be proved by induction on $k$.

For $v, u, u^{\prime} \in I \cup I^{\prime} \cup I^{(1)} \cup \cdots \cup I^{(n)}$ we define \#(v):= the degree of $y$ in $v$, and we say that $u \leq u^{\prime}$ if whenever $u$ has a $y$ in a given position so does $u^{\prime}$. Define $u \cup u^{\prime}$ to be the word that has a $y$ in a given position iff $u$ or $u^{\prime}$ does (e.g., if $u=x x y x y y$ and $u^{\prime}=y x y x x x$, then $u \cup u^{\prime}=y x y x y y$ ). From the definition we have that if $u \leq t$ and $u^{\prime} \leq t$ then $u \cup u^{\prime} \leq t$ and $\#\left(u \cup u^{\prime}\right) \leq \#(t)$.

Next let us consider $\beta_{n-1}$. Since $f_{1}$ is obtained by setting one $x$ equal to $y$ in $p^{\prime}$, the coefficient of $M_{w}$ in $f_{1}$ is

$$
\gamma_{w}=\sum_{j \cdot w=n-1} r_{j}=\beta_{n-1}
$$

Suppose $\beta_{n-1}, \beta_{n-2}, \ldots, \beta_{n-m}$ are the coefficients of $M_{w}$ in $f_{1}, f_{2}, \ldots, f_{m}$, respectively. Now for $\beta_{n-m-1}$, putting $m+1 x$ 's to be $y$ in $\Delta^{1}(y, x) p$ yields the polynomial $\Delta^{m+1}(x, y) \Delta^{1}(y, x) p$, then putting $m$ elements $y$ 's to be $x$ in $\Delta^{m+1}(x, y) \Delta^{1}(y, x) p$ we get the polynomial $\Delta^{m}(y, x) \Delta^{m+1}(x, y) \Delta^{1}(y, x) p$. In $\Delta^{m+1}(x, y) \Delta^{1}(y, x) p$, the monomial $M_{t}$ has coefficient $\lambda_{t}=\sum_{j \cdot t=n-1} r_{j}$, since $\#(j)=n-1$ and $\#(t)=n+m=(n-1)+(m+1)$. (If $j \cdot t<n-1$ then putting $m+1 x$ 's to be $y$ in $M_{j}$ cannot yield $M_{t}$, which has $n+m y$ 's). Similarly, in $\Delta^{m}(y, x) \Delta^{m+1}(x, y) \Delta^{1}(y, x) p, M_{w}$ has coefficient $\mu_{w}=\sum_{t \in I^{(m)}, t \cdot w=n} \lambda_{t}$, since $\#(t)=n+m$ and $\#(w)=n$. Thus

$$
\mu_{w}=\sum_{t \in I^{(m)}, t \cdot w=n}\left(\sum_{j \in I^{\prime}, j \cdot t=n-1} r_{j}\right) \text {. }
$$

We claim that

$$
\begin{aligned}
& \left\{j \in I^{\prime} \mid j \cdot t=n-1 \text { for some } t \in I^{(m)} \text { and } t \cdot w=n\right\} \\
& \quad=\left\{j \in I^{\prime} \mid n-m-1 \leq j \cdot w \leq n-1\right\} .
\end{aligned}
$$

For if $j \in I^{\prime}$ with $j \cdot t=n-1, \#(t)=n+m$, and $t \cdot w=n$, then $j \leq t$, $w \leq t$, and $j \cup w \leq t$. Since \# $(j)=n-1, j \cdot w \leq n-1$. If $j \cdot w \leq n-m-2$ then $j \cup w \leq t$ implies that \# $(j \cup w) \leq n+m$. But

$$
\#(j \cup w)=n-1+(n-j \cdot w) \geq n-1+n-n+m+2=n+m+1,
$$


a contradiction. On the other hand, if $j \cdot w=n-k, k=1,2, \ldots, m+1$, then

$$
\#(j \cup w)=n-1+n-(n-k)=n-1+k \leq n+m ;
$$

therefore, there exists a word $t \in I^{(m)}$ such that $j \cup w \leq t$. Since $j \leq t$ and $w \leq t, j \cdot t=\#(j)=n-1$ and $w \cdot t=\#(w)=n$. This establishes $(*)$. It also means that $r_{j}$ occurs in (6) iff $j \cdot w=n-k$ for some $k$ with $1 \leq k \leq n-m-1$.

Each $r_{j}$ may occur several times in (6). We group the $r_{j}$ 's in (6) depending on $j \cdot w=n-k$ for each $k$. We claim that

$$
\begin{aligned}
& \text { each } r_{j} \text { with } j \in I^{\prime} \text { satisfying } j \cdot w=n-k \text { occurs }\left(\begin{array}{c}
n-k+1 \\
m-k+1
\end{array}\right) \\
& \text { times in (6). }
\end{aligned}
$$

Indeed, the number of the occurrences of $r_{j}$ in (6) is exactly the number of words $t \in I^{(m)}$ such that $t \cdot w=n$ and $j \cdot t=n-1$; that is, $t \in I^{(m)}$ such that $w \cup j \leq t$ since \# $(j)=n-1$. Now if $w \cdot j=n-k$ then

$$
\#(w \cup j)=n+n-1-n+k=n+k-1 \text {. }
$$

So the word $t$ obtained by changing $m-k+1 \quad x$ 's in $w \cup j$ to be $y$ 's belongs to $I^{(m)}$ and $w \cup j \leq t$; however, the number of choices for such a word $t$ is $2 n-(n+k-1)$ choose $m-k+1$, that is, $\left(\begin{array}{c}n-k+1 \\ m-k+1\end{array}\right)$. Hence $(* *)$ holds and

$$
\begin{aligned}
\mu_{w}=\sum_{t \cdot w=n} \sum_{j \cdot t=n-1} r_{j}= & \left(\begin{array}{c}
n \\
m
\end{array}\right) \sum_{j \cdot w=n-1} r_{j}+\left(\begin{array}{c}
n-1 \\
m-1
\end{array}\right) \sum_{j \cdot w=n-2} r_{j} \\
& +\cdots+\left(\begin{array}{c}
n-m+1 \\
1
\end{array}\right) \sum_{j \cdot w=n-m} r_{j}+\sum_{j \cdot w=n-m-1} r_{j} .
\end{aligned}
$$

Since $\beta_{n-k}=\sum_{j \cdot w=n-k} r_{j}$,

$$
\beta_{n-m-1}=\mu_{w}-\left(\begin{array}{c}
n \\
m
\end{array}\right) \beta_{n-1}-\left(\begin{array}{c}
n-1 \\
m-1
\end{array}\right) \beta_{n-2}-\cdots-\left(\begin{array}{c}
n-m-1 \\
1
\end{array}\right) \beta_{n-m} .
$$

Thus, by the induction hypothesis and the definition of $f_{m+1}, \beta_{n-m-1}$ is the coefficient of $M_{w}$ in $f_{m+1}$. Therefore, $\sum \alpha_{k} \beta_{k}$ is the coefficient of $M_{w}$ in $\tilde{p}_{1}$, from the definition of $\tilde{p}_{1}$.

We also know that $c_{w^{\prime}}$ and $(-1)^{n} c_{w}$ are the coefficients of $M_{w}$ in $p(\ldots, y, \ldots, x, \ldots)$ and $(-1)^{n} p(\ldots, x, \ldots, y, \ldots)$, respectively. Thus $(3)$ holds. So statements (1) and (3) are proved. The second statement of the theorem follows easily from $(3)$.

If $\Delta(y, x) p=0$ then $\tilde{p}_{1}=0$ by the definition of $\tilde{p}_{1}$. Hence $p_{1}=0$, which implies that $p$ is symmetric or skew symmetric in $x$ and $y$ depending on whether $n$ is even or odd.

Since the associativity of $F[X]$ was not used in the proof of Theorem 1, Theorem 1 is also true for the free nonassociative algebra. The proof is exactly the same except for the definition of the mapping from $[M]$ to $I$. In this case, $[M]$ consists of monomials having exactly the same distribution of parentheses and that differ only in the positions of $x$ and $y$. When all variables other than $x$ and $y$ are set equal to 1 , a monomial in $x, y$ is obtained. Since the parentheses distribution is always the same, we may therefore drop the parentheses and thus obtain an element of $I$. So again there is a bijection from $[M]$ to $I$. Thus we have 
Theorem $1^{\prime}$. Let $n$ be a positive integer. Let $p$ be a homogeneous polynomial $F\langle X\rangle$, the free nonassociative algebra in the variables $X=\left\{x_{1}, x_{2}, \ldots\right\}$ over a field $F$ of characteristic not dividing $n$ !. Let $x$ and $y$ be arguments of degree $n$. Define $p_{1}$ by

$$
p_{1}:=p(\ldots, y, \ldots, x, \ldots)-(-1)^{n} p(\ldots, x, \ldots, y, \ldots) .
$$

Then the following statements hold:

(1) The polynomial $p_{1}$ comes from a polynomial of lower type than $p$.

(2) Let char $F \neq 2$. If there exist two variables in $p$ of even degree in which $p$ is skew symmetric or of odd degree in which $p$ is symmetric, then $p=\frac{1}{2} p_{1}$ comes from a polynomial of lower type.

(3) If $|F|>2 n-1$ and $p$ is an identity, then $p_{1}$ is a linear combination of identities of type lower than $p$.

(4) If $\Delta(y, x) p=0$ then $p$ is symmetric or skew symmetric in $x$ and $y$ depending on whether $n$ is even or odd.

From Theorem 1' we have

Corollary 1 [Osborn's Theorem]. Let $p$ be a homogeneous identity in a nonassociative algebra $A$ over a field $F$ of characteristic not dividing $n$ !. If $A$ has no identity of type lower than $p$ then $p$ is symmetric or skew symmetric in its arguments of degree $n$, depending on whether $n$ is even or odd.

Theorem 1 holds for varieties of nonassociative algebras. Given an element $p$ in the free algebra of the variety, consider $p$ as an element of the free nonassociative algebra. Equation (3) holds in the free nonassociative algebra and, passing to the quotient algebra, it holds also in the free algebra of the variety.

Theorem 1 says that for each pair or arguments $x, y$ of degree $n$ there exists a polynomial $p^{\prime}=\frac{1}{2}\left(p(\ldots, x, \ldots, y, \ldots)-(-1)^{n} p(\ldots, y, \ldots, x, \ldots)\right)$ that comes from polynomials of lower type such that $p-p^{\prime}$ is symmetric or skew symmetric in $x, y$ depending on whether $n$ is even or odd. In fact, we can find a polynomial $p_{1}$ that comes from some polynomials of type lower than that of $p$ such that $p-p_{1}$ is symmetric or skew symmetric in all its arguments of degree $n$, depending on whether $n$ is even or odd.

Theorem 2. Let $r$ be an integer and $F$ be a field with $\operatorname{char} F \nmid r$ !. Let $V$ be an $\mathscr{S}_{r}$-module. Then

1. $V=\left\{v \in V \mid \pi v=v, \forall \pi \in \mathscr{S}_{r}\right\}+\left(\sum_{1 \leq i<j \leq r}\{v \in V \mid(i, j) v=-v\}\right)$;

2. $V=\left\{v \in V \mid \pi v=-v, \forall \pi \in \mathscr{S}_{r}\right\}+\left(\sum_{1 \leq i<j \leq r}\{v \in V \mid(i, j) v=v\}\right)$.

Proof. To prove the theorem we use induction on $r$. In what follows, we agree that the product of two permutations is performed from right to left. For $r=1$, the theorem holds trivially. For $r=2$, every $v \in V$ has a unique decomposition

$$
v=\frac{1}{2}(v+(12) v)+\frac{1}{2}(v-(12) v) .
$$

Suppose the result is true for $r-1$. We imbed $\mathscr{S}_{r-1}$ into $\mathscr{S}_{r}$ as $\left\{\pi \in S_{r} \mid \pi(r)=\right.$ $r\}$. Thus a given $\mathscr{S}_{r}$-module is also an $\mathscr{S}_{r-1}$-module. Hence by induction,

$$
V=\left\{v \in V \mid \pi v=v, \forall \pi \in \mathscr{S}_{r-1}\right\}+\left(\sum_{1 \leq i<j \leq r-1}\{v \in V \mid(i, j) v=-v\}\right) .
$$


Let $v \in V^{\mathscr{S}_{r-1}}:=\left\{v \in V \mid \pi v=v, \forall \pi \in \mathscr{S}_{r-1}\right\}$ and define

$$
w:=v-\frac{1}{r} \sum_{1 \leq i \leq r-1}(v-(i r) v)
$$

It suffices to show $w \in V^{\mathscr{S}_{r}}$. We have

$$
w=\frac{1}{r}\left(v+\sum_{1 \leq i \leq r-1}(i r) v\right),
$$

hence for $1 \leq j \leq r-1$,

$$
(j r) w=\frac{1}{r}\left[(j r) v+v+\sum_{1 \leq i \leq r-1, i \neq j}(j r)(i r) v\right]=w,
$$

since $(j r)(i r)=(i r)(i j)$, and thus

$$
(j r)(i r) v=(i r)(i j) v=(i r) v,
$$

using $v \in V^{\mathscr{S}_{r-1}}$. Because $\{(j r) \mid 1 \leq j \leq r-1\}$ generates $\mathscr{S}_{r}$, we have shown that $w \in V^{\mathscr{S}_{r}}$, completing the proof of Theorem 2(1).

Symmetrically, to prove Theorem 2(2) we define

$$
w^{\prime}:=v^{\prime}-\frac{1}{r} \sum_{1 \leq i \leq r-1}\left(v^{\prime}+(i r) v^{\prime}\right),
$$

where $v^{\prime} \in\left\{v \in V \mid \pi v=-v, \forall \pi \in \mathscr{S}_{r-1}\right\}$. Now we can repeat the proof of (1) of Theorem 2 step-by-step to show that $w^{\prime} \in\left\{v \in V \mid \pi v=-v, \forall \pi \in \mathscr{S}_{r}\right\}$. This completes the proof of Theorem 2.

Let $V=V^{\left[m^{r}, n^{s}, \ldots, u^{t}\right]}[X]$, and

$$
W_{1}=\operatorname{Span}\left\{f\left(x_{1}, \ldots, x_{r}, y_{1}, \ldots, y_{s}, \ldots\right) \in V \mid\right.
$$

$f$ is skew symmetric in $x_{i}$ and $x_{j}$ for some $\left.i \neq j\right\}$.

Let $U$ be the set of the element $f\left(x_{1}, \ldots, x_{r}, y_{1}, \ldots, y_{s}, \ldots, z_{1}, \ldots, z_{t}\right) \in$ $V$ that are symmetric or skew symmetric in some $w_{i}, w_{j}$ where $i \neq j$ and $w \in\{x, y, \ldots, z\}$, depending on whether the degree of $w_{i}$ in $f$ is odd or even. Let $V_{1}=\operatorname{Span} U$. Then we have

Theorem 3. Let $m_{0}=\max \{m, n, \ldots, u, r, s, \ldots, t\}$. If $\operatorname{char} F \nmid m_{0}$ ! then every element $f$ in $V$ has a decomposition into a sum of two polynomials of the same type as $f, f=f_{0}+f_{1}$, where for each $k$ with $0<k \leq m_{0}, f_{0}$ is symmetric or skew symmetric in all variables of degree $k$ depending on whether $k$ is even or odd and $f_{1} \in V_{1}$. Moreover $V_{1}$ is spanned by elements that come from lower type polynomials: if $g \in V_{1}$ then $g=\sum g_{k}$, where $g_{k} \in U$ and $g_{k}$ is given by formula (2).

Proof. Let $f\left(x_{1}, \ldots, x_{r}, y_{1}, \ldots, y_{s}, \ldots, z_{1}, \ldots, z_{t}\right)$ in $V$. First we show that $f=f_{0}+f_{1}$, where $f_{1} \in V_{1}$ and $f_{0}$ is symmetric or skew symmetric in all $x_{i}$ 's depending on whether $m$ is even or odd. For an arbitrary $\sigma \in \mathscr{S}_{r}$ we define

$$
\begin{aligned}
& \sigma\left(f\left(x_{1}, \ldots, x_{r}, y_{1}, \ldots, y_{s}, \ldots, z_{1}, \ldots, z_{t}\right)\right) \\
& \quad:=f\left(x_{\sigma(1)}, \ldots, x_{\sigma(r)}, y_{1}, \ldots, y_{s}, \ldots, z_{1}, \ldots, z_{t}\right) .
\end{aligned}
$$


Then $V$ is an $\mathscr{S}_{r}$-module. If $m$ is even then by Theorem $2(1), f=f_{0}+$ $f_{1}$ where $f_{1} \in W_{1}$ and $f_{0}$ is symmetric in all $x_{i}$ 's. Since $W_{1} \subseteq V_{1}$ by the definitions of $W_{1}$ and $V_{1}$, we get $f_{1} \in V_{1}$. If $m$ is odd then we use Theorem 2(2).

In any case, we have that $f=h_{0}+h_{1}$, where $h_{1} \in V_{1}$ and $h_{0}$ is symmetric or skew symmetric in all $x_{i}$ 's depending on whether $m$ is even or odd. Next we consider $h_{0}$ and variables $y_{1}, \ldots, y_{s}$ as above. The above process will not destroy the symmetry of $h_{0}$ in the $x_{i}$ 's. Thus after a finite number of steps, we have that $f=f_{0}+f_{1}$, where $f_{1} \in V_{1}$ and $f_{0}$ is as in the theorem.

The last statement of the theorem follows from Theorem 1 and the definition of $U$.

To summarize, if $f \in T(A, W) \cap V$ then $f=f_{0}+f_{1}$ with $f_{i}$ an identity, for $i=0,1$ by Theorem 3 and the fact that $T(A, W) \cap V$ is an $\mathscr{S}_{r}$-module. But we do not know whether $f_{1}$ comes from the identity of lower type although it comes from a lower type polynomial. However, using Proposition 1 we have

Corollary 2. Let $m_{0}=\max \{m, n, \ldots, u, r, s, \ldots, t\}$. If char $F \nmid m_{0}$ ! and $|F|>2 m_{0}-1$, then every identity $f$ in $V$ has a decomposition into a sum of two identities of the same type as $f, f=f_{0}+f_{1}$, where for each $k$ with $0<k \leq m_{0}, f_{0}$ is symmetric or skew symmetric in all variables of degree $k$ depending on whether $k$ is even or odd and $f_{1}$ comes from lower type identities.

\section{ACKNOWLEDGMENTS}

The results of this paper are part of the author's dissertation at the University of Ottawa. The author is grateful to his advisor Professor M. L. Racine and to Professor E. Neher for their helpful suggestions and comments. The author would also like to thank Dr. Alain D'Amour for his help in reading and criticising the first version of the paper.

\section{REFERENCES}

1. Wenxin Ma and Michel L. Racine, Minimal identities of symmetric matrices, Trans. Amer. Math. Soc. 320 (1990), 171-192.

2. J. Marshall Osborn, Identities of nonassociative algebras, Canad. J. Math. 17 (1963), 78-92.

3. K. A. Zhevlakov, A. M. Slin'ko, I. P. Shestakov, and A. I. Shirshov, Rings that are nearly associative, Academic Press, New York, 1982.

Department of Mathematics, University of Ottawa, Ottawa, Ontario, Canada K1N 6N5 